\title{
Analysis of Human Errors in Industrial Incidents and Accidents for Improvement of Work Safety
}

Leplat, J.; Rasmussen, Jens

Published in:

Accident Analysis \& Prevention

Publication date:

1984

Document Version

Peer reviewed version

Link back to DTU Orbit

Citation (APA):

Leplat, J., \& Rasmussen, J. (1984). Analysis of Human Errors in Industrial Incidents and Accidents for Improvement of Work Safety. Accident Analysis \& Prevention, 16(2), 77-88.

\section{General rights}

Copyright and moral rights for the publications made accessible in the public portal are retained by the authors and/or other copyright owners and it is a condition of accessing publications that users recognise and abide by the legal requirements associated with these rights.

- Users may download and print one copy of any publication from the public portal for the purpose of private study or research.

- You may not further distribute the material or use it for any profit-making activity or commercial gain

- You may freely distribute the URL identifying the publication in the public portal

If you believe that this document breaches copyright please contact us providing details, and we will remove access to the work immediately and investigate your claim. 


\title{
ANALYSIS OF HUMAN ERRORS IN INDUSTRIAL INCIDENTS AND ACCIDENTS FOR IMPROVEMENT OF WORK SAFETY ${ }^{1}$
}

\author{
Jacques Leplat \\ Laboratoire De Psychologie Du Travail, F 75005 Paris, France \\ and \\ Jens Rasmussen \\ Risø National Laboratory, DK 4000 Roskilde, Denmark
}

\begin{abstract}
Methods for the analysis of work accidents are discussed, and a description is given of the use of a causal situation analysis in terms of a "variation tree" in order to explain the course of events of the individual cases and to identify possible improvements. The difficulties in identifying "causes" of accidents are discussed, and it is proposed to analyse accident reports with the specific aim of identifying the potential for future improvements rather than causes of past events. In contrast to traditional statistical analysis of work accident data, which typically give very general recommendations, the method proposed identifies very explicit countermeasures. Improvements require a change in human decisions during equipment design, work planning, or the execution itself. The use of a model of human behaviour drawing a distinction between automated skill-based behaviour, rule-based "know-how" and knowledge-based analysis is proposed for identification of the human decisions which are most sensitive to improvements.
\end{abstract}

\section{INTRODUCTION}

In different branches of industry, different methods have been developed for analysis of incidents and accidents in order to identify their causes and propose improvements for safety. Within process industries characterised by large production units and high levels of automation, risk and accident analysis is focused on avoidance of low probability events with large consequences to the plant and its environment. Analysis is here based on causal models of the accidental chains of events which can serve to identify deficiencies in the design of the plant and its protective systems, as well as to predict the level of risk involved in operation. Detailed analysis of the actual, individual incidents and accidents is normally performed to identify these possible weak spots in the plant and its operation. It is a general experience that human acts play an important role in such industrial accidents, and much effort is spent to include human performance in the causal accident models. This approach is feasible because of the well structured nature of industrial process plants,

${ }^{1}$ Accid. Anal. and Prev. Vol. 16, No. 2, pp.77-88, Pergamon Press Ltd., 1984. (Received 4 April 1983; in revised form 15 July 1983) 
which also leads to a well defined task structure for the operators involved.

The situation is quite different for safety aspects related to workshops and construction sites, characterised by much less structured work situations and a high number of reported cases. Consequently, analysis is typically based on statistical and epidemiological methods rather than detailed causal analyses of the individual cases. Such analyses are generally based on an analysis of the routine reports collected by safety authorities which give only sparse information on the individual events and, in particular, on the work conditions and the normal routines which led to the case [Leplat, 1978, 1982]. Typically, such data are used for management and insurance purposes, rather than design of countermeasures.

During recent years, several attempts have been made to use the causal situation analysis on work accidents; a promising approach has been the "I.N.R.S. method" described in the following section. However, the aim of this method as well as other attempts have often been to explain the individual cases; to identify the causes of the accidents.

The present paper suggests an analysis which focuses on the identification of points in the sequence which are sensitive to future improvements rather than causes of past events. It is based on the "analysis of variations" developed for analysis of work accidents, combined with a model of human decision making based on analysis of human performance and errors in process plants.

\section{ANALYSIS OF INCIDENTS/ACCIDENTS}

In this first part, a method which is useful for bringing some light upon the causation of incidents and accidents is described. Reliability specialists have long been interested in this problem and typically will use fault tree analysis in order "to model the system conditions that can result in the undesired event" (. . . ) "A fault tree is a model that graphically and logically represents the various combinations of possible events, both faulty and normal, occurring in a system that leads to the top event" [Barlow and Lambert, 1975, p. 8]. In the present context, the top event will be the incident or accident. The application of this method presupposes a number of conditions, typically related to the well-defined structure of process plants and other technical machinery, which are rarely found in socio-technical systems. The method presented here is certainly less systematic, but its possibilities of use are much broader, for instance for study of work accidents in workshops and construction sites. In particular, 
when used for post hoc analysis, it differs from the fault tree on one specific point: the top event is not a class of events but one particular event-incident or accident-which actually occurred and for which the antecedent chain of events must be discovered. The tree obtained is, formally, a sub-tree of the fault tree (which would include a family of possible chains of events leading to the same type of accident). This subtree is peculiar in having no "OR gates". Combining a number of such sub-trees for a given type of accident would lead to a fault tree, defined after the fact.

\section{(a) The method of variation tree ${ }^{1}$}

This method is based on the idea that the occurrence of an accident results from aberrations or variations happening in the normal or "habitual" work situation. During the analysis of an incident or accident, it is first necessary to identify such changes; i.e. to establish a list of changes and then to organize them in order to define their interrelationship. The two most important relations are the following:

The event-chain relationship, noted $X->Y$. Indicates that event $X$ is a necessary condition for the occurrence of event $Y$, or that without event $\mathrm{X}$, event $\mathrm{Y}$ would not have occurred.

The confluence relationship, noted $X 1, X 2->Y$. Indicates that the simultaneous presence of several (here two) independent events or states is necessary for the occurrence of event $Y$. It can be expressed: "without event $\mathrm{Xl}$ or $\mathrm{X} 2$, event $\mathrm{Y}$ would not have occurred". Of course other Xi may be necessary for the occurrence of $\mathrm{Y}$; they are not mentioned because they are not "variations".

This method can be illustrated by a simple diagram of an accident on a drilling machine.

Remarks

(I) To build this diagram, it is necessary to know the activity (in more or less detail, depending on the case) in order to define the variations and the relations between them. Let us consider for example the relation $\mathrm{X} \sim \mathrm{Y}$. Y can depend on $\mathrm{X}$ through a physical necessity, e.g. the clothing must be in contact with the drill in order to be caught. $\mathrm{Y}$ can depend on $\mathrm{X}$ due to operating rules and practice. The machine is set in motion, e.g. because the operator manipulates the starting button. $\mathrm{Y}$ can depend on $\mathrm{X}$ due to

\footnotetext{
${ }^{1}$ This method, which was designed originally by Cuny and researchers from I.N.R.S, National Institute for Research and Safety), is rather widespread in France with the name "I.N.R.S. method" or more properly) "method of tree of causes". In English, a more detailed statement and example can be found in papers from Cuny, Leplat Quinot in "Research on Occupational Accidents", 1977, Arbetarskyddfonden, Stockholm. In French, numerous examples are in the reports of 1.N.R.S. (Institut National de Recherche et de Securite pour la Prevention des Accidents du Travail et des Maladies Professionnelles-30, rue Olivier, Noyer, 75680 Pans Cedex 14).
} 
organizational rules, e.g. the machine is set in motion because an order is given by the supervisor. One must add "etc." to this list. The existence of many relations depends on the subjective perception of relations by the operator: relations may therefore depend on the individual operator and be difficult to identify after the fact.

(2) The diagram of variations constitutes only a part of the generic diagram representing causation. When one reads $\mathrm{X} \sim \mathrm{Y}$, one must be aware of translating " $\mathrm{X}$ entails $\mathrm{Y}$ " because $\mathrm{X}$ is a necessary but, however, not a sufficient condition to produce $Y$. It will sometimes be helpful to introduce in the diagram of variations a number of normal operating states or events in order to have a greater insight into the causal mechanisms and, thus, of possible preventive measures. For instance, on the diagram of Fig. I, a branch can be added to the conjunction which would be "drill is rotating". It would permit a better understanding of the final event. Therefore, the diagram of variations should be considered as a support to the analysis of causation of an accident.

(3) The variations can be classified in several categories. The I.N.R.S. method classifies them according to the following socio-technical categories [Krawsky et al., 1972]: I (individual), $\mathrm{T}$ (task), M (machine), E (environment). In the method of analysis presented in the following paragraphs, we distinguish between the states of the system or its elements, and the events (or actions) which are changes of the states. We are here concerned particularly with the individual and his actions.

\section{(b) An example-a driving accident}

To illustrate the diagram of variations and to have a case for further discussion in subsequent sections, the following accident is considered.

A lorry driver is ordered to deliver a cargo at a certain destination. As his usual lorry has had a break-down, he picks up a replacement lorry, which is unfamiliar to him and, as it turns out, has faulty brakes. The lorry is then loaded and in fact overloaded. On route it appears that the route the driver usually takes is closed due to road repair. The driver takes a detour which turns out to have an unexpected steep slope. Due to slope, load, and bad brakes, the driver loses control and is unable to follow the curve in the road. He runs he lorry into a wall and is severely injured. The diagram of variations is shown on Fig. 2. 


\section{(c) The combination of the diagrams of variations}

When a number of incidents or accidents have been analysed on the same system, for example on the same type of machine, we can try to merge the corresponding diagrams of variations. This has been done by Bruun et al. [1979] by means of cause-consequence charts, proposed by Nielsen 1975]. Figure 3 gives the main elements of such a diagram. Figure 4 shows the principle of the combination of diagrams from a simple example. Bruun et al. [1979] give more complete examples obtained from a set of accidents which occurred on drilling machines.

In order that a set of diagrams can be merged, a number of conditions of homogeneity must be met which are expressed by Bruun et al. [1979, p. 7$]$ as follows:

- Choose the same starting point for analysis of similar accidents".

- "Choose the same level of detail for recording each accident".

-"Trace the causes of accidents backwards to the same stage in causation".

- Use the same terms at each stage in the accident descriptions to describe identical events or conditions".

One can add "Have a consistent concept of variation". To merge the diagrams, it will sometimes be necessary to complete them with elements which are not variations (but normal states, activities, or events). The formation of merged (or synthetic) diagrams permits the evaluation of the risk presented by some characteristics of the operator and machine (from the frequency of incidents and accidents) and the identification of critical routes leading to the accident: in consequence, its usefulness for preventive safety measures is quite clear.

\section{ANALYSIS OF HUMAN ERRORS}

The analysis of human errors and their role in industrial accidents is an important part of the development of systematic methods for industrial reliability and risk prediction. In order to obtain data for such predictive analyses, for instance by means of fault trees, as previously mentioned, it is necessary to analyse accidents and incidents to identify their causes in terms of component failures and human errors, since data on the frequency of such causes will make it possible to estimate the probability of the related chains of events during design of new systems.

However, the determination of how far back in an accidental chain of events it is necessary to go in order to find its causes is a matter of 
practical judgement and depends on the purpose of the analysis. Typically, if a component failure is identified at a level where the component is easily replaced, this component failure is taken as the cause if no special circumstances make it necessary to trace further back to identify causes in terms of production or design deficiencies. Definition of human errors is even more ambiguous, since the human typically will notice inappropriate actions and correct them before they lead to unacceptable consequences. Therefore, whether a given inappropriate act will count as an error and contribute to the frequency of causes depends as much upon the characteristics of the work environment in terms of immediate error observability and reversibility as it depends on human error frequencies.

A more fruitful point of view is to consider human errors as instances of man-machine misfits, i.e. instances when human variability is not within the span acceptable for successful task performance [Rasmussen, 1981]. Variations in performance become human errors only in an "unkind" environment which does not allow immediate correction. This means that to characterize human "errors", one has to determine the variability of human behaviour and the acceptance limits for variation which hold for the work situation.

Generally, human errors are defined in terms of the faulty, external task element and data are collected correspondingly. If, however, the point of view of variability of human behaviour is taken, it is necessary to identify the varying element of human behaviour in terms referring to the internal human properties.

There is no one-to-one relationship between the external task performance and the internal human functions which are used. Consequently, to characterize the human role in an accidental chain of events, it is necessary also to consider the internal mental decision functions which are required in the task (Fig. 6), the related internal psychological mechanisms which are involved in the error (Fig. 5), and the possible presence of external causes to the particular human response. The internal decision functions and error mechanisms depend on the psychological functions and level of knowledge which are activated by subjective factors in a particular situation. They cannot be directly observed but must be inferred from characteristics of the task and the work situation together with the external manifestation of the error. For this to be possible, a model of human information processing must be available. Such a model must relate elements of human decision making and action to internal information processes for which generic psychological mechanisms and limitations can be identified. An attempt to develop such a model from analysis of verbal protocols and cases of human malfunction has 
been described elsewhere [Rasmussen, 1976,1980]. In this model, a distinction is drawn between three levels of behaviour: skill-, rule-, and knowledge-based performance, see Fig. 5. The figure illustrates how different internal mechanisms are used for the same decision function, depending upon the degree of familiarity with the work situation and task element.

The use of such a model in the design of a multi-facet taxonomy for human errors to be used for data collection by analysis of event reports has been proposed elsewhere [Rasmussen, 1981]. For reliability prediction, this approach may be useful for the design of man-machine interface systems for industrial process plants. The highly structured functional properties of such systems lead to a fairly well defined anatomy of accidents, in which causes and latent failure conditions can be reasonably well defined and identified by post hoc analysis.

For less structured work situations and hence for the study of common work accidents, this approach is not immediately attractive, since the causal net and its topology are not so well defined, and the reference situations used to define errors and causes are therefore questionable. It appears, however, that a promising approach can be found if the analysis of accidents is oriented more to the identification of future improvements than towards past causes. This will be discussed in more detail in the following section.

\section{ANALYSIS OF ACCIDENTS FOR POINTS OF CRITICAL DECISIONS}

A common feature of the two approaches to the analysis of accidents and incidents discussed in the two preceding sections is the view of such events as the consequences of chains of events released and/or conditioned by a number of "variations" with respect to a normally successful performance. It appeared to us that this view, if carried further in its consequences, could lead to a more fruitful approach to the post hoc analysis of accidents. Rather than identifying causes of accidents in order to collect data for their frequency or to find means for their removal-or, as is frequently the case, to find someone to blame-it appears to be more appropriate to map an accident as a tree of variations and to look for the effect of variations towards safety which might be introduced. It may be much more fruitful to introduce a variation that will break the accidental sequence than to try to remove its cause(s). In the following we discuss this approach in more detail. In effect, it amounts to finding critical decision points where conditions for existing decisions or acts can be improved or new deci- 
sions can be inserted to change the route of events in a safe direction.

The basis for the analysis will be a variation diagram, as discussed in Section II. This diagram should be prepared very carefully in order to identify "variation" antecedents to the accidental event as well as "state" antecedents. State antecedents are states which act by conditioning the work context and therefore prepare the route of events. They are very likely.latent effects of previous variations with respect to a safe condition, since the nodes of the diagram are typically variations from normal.

What kind of changes can be introduced to break the flow of events in the variation diagram? It appears immediately that the course of events is broken if the conditions producing a variation are removed or the effects cancelled. However, since the release condition for one node of variation is the effect of an antecedent variation, it appears that a general rule will be to cancel a variation node, either by changing the physical condition (replace a worn tyre on a car), or changing the basis for a human act (by training, better information in the situation, or more tolerant work conditions). In case of confluence relationships, it suffices to cancel one of the necessary events (see Fig. I) or state antecedents. In other words, the problem is to identify those changes or improvements which will eliminate the effect of human variability during the course of events, or will counteract prior variations or decisions, for instance during the design phase, which lead to antecedent states preparing the route of the accident.

Another possibility will be to break the.flow of events between nodes in the diagram of variations. What change can sensibly be introduced that will activate a human to make a decision in the sequence which breaks or corrects the flow of events? How can the mental state of humans or the work condition be changed so as to lead a person involved to detect and correct the risky conditions?

To develop this approach into a practical method, at least two major problems should be considered.

First, considering a single accident case, it is very easy to suggest many changes ad hoc which would break the sequence. Therefore a screening method must be found to select the reasonable candidates. One such method could be to superimpose a number of variation diagrams with suggestions for changes, derived for similar accident sequences or similar work conditions, in order to identify changes which have recurrent effect, and thus reject ad hoc candidates which are only relevant for a single case.

It is by no means a new idea to try to identify factors which can be changed in order to break accident sequences. However, this is typically done by generalizing into a few categories of improvement 
during the analysis of the individual cases, or by making correlation analyses between accident statistics and a number of general factors as level of education, weather conditions, etc. Typically, this analysis is performed as standard statistical analysis directly on the data contained in the accident reports collected by work safety authorities. In practice this leads to very general recommendations like better education, more effective maintenance, etc., which are very difficult to implement or make operational. In the approach proposed here, the changes are identified very explicitly and in detail for every case, and generalisation is only made after a high number of cases have been merged. This makes the identified changes very specific and operational. However, this kind of analysis cannot be undertaken in the same way as standard statistical analyses of the data in accident reports. It is necessary to convert the data in routine reports into diagrams of variations; i.e. the accident reports should be related to knowledge of the normal, successful performance. This means that information should be added to the reports which is only available from persons intimately familiar with the normal work routine, as for instance supervisors or work safety representatives on site.

Second, we have to identify the changes in work conditions or in human disposition which will cause the proper decisions to be made. It is a general observation that motivation programs or request that people should "try harder" have limited effect. This problem will be discussed in more detail in relation to the human performance model, but first we have to identify the kind of decisions we want to influence.

In general, analysis of accidents and the resulting diagram of variations will be expressed as a sequence or tree including observable physical events or human acts. In order to identify means for breaking the sequence we will be looking for nodes in the diagram which are sensitive to cancelling. For nodes representing inappropriate acts, this must be done by improving conditions for human decision and acting during the situation. For nodes representing abnormal states of the physical work environment, cancelling involves improvement of this physical condition, i.e. the conditions for decision making during work planning or system design must be evaluated for possible improvements.

Looking for suitable means for breaking the sequence by removing the causal relation among nodes, we can also look for reasonable points when people during the event can be made aware of the risky course of events and enabled to make decisions which can break the sequence or change its course. In addition, we can look for suitable locations for physical changes which will act as barriers against the 
flow of events. This means to look for suitable decision points during work or system design.

It will be seen that the analysis focuses the attention on decisions related to the variations at all levels in an organisation, not only the worker directly involved. For overloading the truck in the example of Fig. 2, not only the loading itself, but also driving schedules, payment policies and supervisory control must be considered.

It will in all cases be necessary to evaluate all phases of the sequence involved in human decision making and acting in order to identify the sensitivity to improvement, i.e. whether support should be given to detection of the course of event under way, to identification of the problem, to evaluation of goals and priorities, or to planning and execution of the intervention. In some cases, just making the person aware that the potential for a risky chain of events is present will activate proper decisions. In other cases, support of proper identification of the circumstances will be vital, sometimes the consideration of conflicting goals must be considered, and so forth.

To be able to judge the appropriate means for changing the decisions of humans involved in the accidental chain of events or the planning of work conditions, it is necessary to consider the level of cognitive awareness of the persons during the situations in which it is planned to change their decisions. The rational decision sequence involving all phases is illustrated in Fig. 6 which also shows how their relevance depends on the level of cognitive activity involved in the performance; i.e. upon the training of the person. During highly trained, skill-based routines, the decision sequence is not at all activated; and during familiar tasks based on know-how, the higher level decision phases by-passed by stereotype rules and habits. The point here is that it will not be reasonable to base improvements on support of decision phases which are in fact by-passed during the actual situation. If an activity is controlled by highly skilled routines, it will be less effective to change it by improving the knowledge of the persons involved or by asking them to be more careful. The most effective influence will be through changes of the work situation. This means that it is necessary to identify a person in the work planning or design phase who will work at a level of cognitive awareness and whose decisions can be affected.

To conclude, we propose that the traditional search for means to improve work safety through epidemiological analysis of accident records based on statistical data and very general characteristics of the work situation should be supplemented by detailed analysis of the diagram of variations for each accident which includes experts' knowledge of the normally successful work performance. Improvements are 
identified for each case separately, carefully considering the level of conscious awareness of the people involved in the specific situations in order to eliminate psychologically infeasible ad hoc proposals. Candidates for serious consideration as proposals for improvements are then identified by an "overlay" of sets of variation diagrams for similar work situations and types of accident in order to have a screening against singular ad hoc proposals. The approach can be illustrated by the following example.

\section{Analysis of driving example, Fig. 2}

First, we consider the possibility of cancelling the nodes of variation in the diagram given in Fig. 2. The phases in the decision sequence which are judged to be sensitive to improvements are indicated in Fig. 7 by the number of node to cancel indicated by ( $n$ ) or path to break indicated by $\mathrm{I}^{\mathrm{n}}$.

\section{Cancelling nodes}

(1), (2) and (3) related to road conditions do not lend themselves to control in the present context. To propose a decreased slope appears to be an ad hoc solution of low generality. (4) overload. Changes towards safety may involve:

-Detection, makes driver alert by some kind of overload alarm.

-Observation, easy access to weight information.

-Evaluation, change criteria for judgement. It will probably be difficult to influence highly skilled people at this level by motivational programs, etc. Therefore, changes involve changes in work condition and social context, i.e. decisions in work planning context related to company policy, payment, penalties, etc.

(5) and (6) faulty truck and replacement. Change the rules of allocation of trucks. (7) inadequate maintenance. Reorganize the maintenance department. (8) brakes faulty:

-Detection; worn brake alarm devices; regular inspection. Evaluation; changing criteria, penalties.

(9), (10) and (11) loss of control. Since the driver at this point is well aware of the problem, cancelling will be related to defining the task to pursue (to save the car/load/people/himself and to find the proper procedures. Since skilled drivers do not depend on conscious planning, verbal instruction will probably be less efficient than real training in emergency procedures. (12) avoid injury. Cancelling here involves introduction of protective devices such as safety belt, absorbent lorry bodies, etc. This involves high level decisions during lorry design. 


\section{Breaking paths}

1. Avoiding steep slope route. Better slope information on route (sign?); better understanding of slope/load relations (probably not feasible for highly skilled driver who will not perform rational evaluations).

2 Driver does not accept lorry with faulty brakes, as (8).

The general rule is that when an improvement can be envisaged involving a certain decision phase, it will be necessary to find a person in the system for whom that phase is active and not normally bypassed by habits and routines. The list given above is meant to be illustrative, not complete. Further screening of ad hoc solutions will be needed by merging several diagrams for traffic accidents with heavy trucks, either for accidents within the company or for accidents in particular areas, depending on the purpose of the analysis.

\section{CONCLUSION}

The present paper proposes the general outline of an approach to the analysis of work accidents with the specific aim of safety improvement, rather than the evaluation and understanding of past events. Further development is needed to evaluate its practical value. For this purpose, a tight cooperation between several professional areas is necessary in the analysis of events. The analysis requires access to a representative number of uniform cases which is only possible for a central safety authority. On the other hand, the analysis requires that the routine event reports are studied by persons with an intimate knowledge of the work situation, the normal work routines and the tools and equipment used, as well as the organisational and socio-technical relationships. This information is available only to persons who participate in the particular industrial work situations and, therefore, can identify "variations". Finally, the analysis requires psychological expertise and knowledge about relevant models of human decision making.

Development of the method therefore requires a mutual and positive cooperation among representatives of industrial workers, authorities and academic research-cooperation which has its own problems and conflicts. From the test cases, however, it is our experience that such a cooperation can be established, and that the approach is worth pursuing.

The approach would serve to improve the skill of the safety officer and, in general, contribute to a better management of reliability and safety in socio-technical systems. In addition, it is worthwhile to emphasize the amenability of the proposed method to further 
improvements as the result of experience.

\section{REFERENCES}

Barlow R. R. and Lambert H. E., Introduction to Fault Tree Analysis in Reliability and Fault Tree Analysis,

(Edited by R. E. Barlow et al.) pp. 7-36. Society for Industrial and Applied Mathematics, 1975.

Bruun 0., Rasmussen A. and Taylor J. R., Cause-Consequence Reporting for Accident Reduction. Risø-M-2206

Krawsky G., Cuny X. and Monteau M., Methode Pratique de Recherche des Facteurs d'Accidents. Rapport No. 24/RE/A, Vandoeuvre, I.N.R.S., France 1972.

Leplat J., Accident analyses and work analyses. J. Occ. Accid 1, 331-340 1978.

Leplat J., Fiabilite et securite. Le Travail Humain 4S, 1, 101-108, 1982.

Rasmussen J., What can be learned from human error reports?. In Changes in Working Life (Edited by Duncan, Gruneberg and Wallis). Wiley, New York, 1980.

Rasmussen J., Human errors. A taxonomy for describing human malfunction in industrial installations. J. Occ. Accid 4, 311-333, 1982. 